\title{
La incidencia de las variables persistentes de la política exterior argentina: el caso de la relación con México
}

María Julia Francés ${ }^{I}$

\begin{abstract}
Resumen: El presente artículo analiza las relaciones entre dos "extremos" geográficos de América Latina, Argentina y México, desde la perspectiva de la política exterior de la primera (PEA). Así, profundizaremos en la noción de latinoamericanismo y comprender hasta qué punto se refleja en hechos reales y constantes, más allá de los vínculos circunstanciales o de vecindad. Para ello, utilizaremos la noción de "variable persistente" para analizar algunos aspectos que consideramos claves en la PEA en general y para este vínculo en particular. El objetivo del trabajo será analizar la incidencia de estas características históricas sobre el caso de la relación argentina con México durante las administraciones de Cristina Fernández. Nos preguntaremos si, con sus conflictos y coincidencias, las características del accionar externo argentino se enmarcan en una verdadera estrategia latinoamericanista más allá de la subregión sudamericana y si los demás aspectos analizados de la PEA inciden positiva o negativamente sobre dicha cuestión. Para ello, haremos especial hincapié en una serie de variables: el economicismo y el juridicismo que conjugaremos con la problemática de los recursos naturales y el territorio, para finalmente enfocarnos en el vínculo con la región y el concepto de latinoamericanismo.
\end{abstract}

Palabras claves: latinoamericanismo, variables persistentes, política exterior argentina.

\section{The influence of Persistent Variables on Argentina's Foreign Policy towards Mexico}

\begin{abstract}
This article studies the relationship between two "opposite ends" of Latin America, Argentina and Mexico, from the point of view of the former's foreign policy. It explores the idea of Latin-Americanism by posing one key question: is this concept reflected on real and consistent actions that go beyond temporary circumstances or mere geographical vicinity? In order to answer this question, there are a series of "persistent variables" in Argentina's foreign policy (AFP) that must be taken into account when analyzing the country's history of relations with the continent. The aim of this paper is to study the influence of these historical traits specifically for the case of the Fernández administrations' policy towards Mexico. The focus is particularly set on whether Buenos Aires has carried out a true and systematic Latin-Americanist strategy beyond its subregion and whether its "persistent variables" have a positive or negative effect on this matter. The AFP variables chosen for this analysis are: economism and juridicism and its link to natural resources and territorial matters, as well as Argentina's relationship with the region as a whole.
\end{abstract}

Key words: Latin-Americanism, persistent variables, Argentine foreign policy

Artigo recebido em 01/04/2016 e aprovado em 07/04/16. 


\section{LA INCIDENCIA DE LAS VARIABLES PERSISTENTES DE LA POLÍTICA EXTERIOR ARGENTINA: EL CASO DE LA RELACIÓN CON MÉXICO}

\section{MARÍA JULIA FRANCÉS}

El presente trabajo se enmarca en las investigaciones realizadas por el Observatorio de Política Exterior Argentina (OPEA), que incluyen tanto informes semanales como artículos de análisis. En estos últimos hemos procurado comenzar a echar luz sobre una temática ampliamente debatida por los especialistas: la idea de "latinoamericanismo" y su aplicabilidad al estudio de la política exterior argentina. Es un concepto polisémico puede también vincularse con expresiones de hermandad latinoamericana, regionalismo latinoamericano e incluso con integración regional. Estas nociones han sido repetidas con frecuencia desde la independencia de las excolonias españolas en el continente y han llevado a diversas conclusiones y perspectivas que influyeron, en mayor o menor medida, en la política exterior de la región a lo largo del tiempo.

Desde las tesis unionistas, antiimperialistas y panamericanistas hasta los regionalismos abiertos más recientes, el vínculo especial entre estos países ha tenido distintas características y se le ha asignado diversas jerarquías dentro de las agendas de política exterior de cada una de estas naciones. Para la autora María Cecilia Zuleta en su libro "Hispanoamérica en sus límites", es de especial interés tratar esta temática haciendo un análisis histórico de su trayectoria desde la independencia de los países involucrados, pero concentrándose en los dos extremos de la región: el Cono Sur y México. Y es que la distancia geográfica y simbólica entre estas dos partes puede indicarnos que, más allá de las coincidencias idiomáticas e históricas, la cercanía entre estas naciones es más una construcción que un hecho histórico ineludible.

Si pensamos que efectivamente existe un sentimiento latinoamericano en los pueblos y en aquellos actores que configuran las relaciones entre ellos, buscamos prueba de ello en las acciones externas de los estados. Y como abarcar el conjunto de los países latinoamericanos a lo largo de su historia excedería las posibilidades del presente capítulo, nos concentramos en el accionar de uno de ellos hacia otro, con una intención similar a la citada autora María Zuleta, es decir, en la política exterior de Argentina (nuestro tema de estudio en el Observatorio) en su relación con México. Los dos extremos geográficos de la región.

La selección de estos socios no es casual, sino que responde a un interés por profundizar algunas hipótesis planteadas en los espacios de análisis del OPEA en relación con la caracterización de la política exterior argentina (PEA) durante las últimas administraciones. Desde un comienzo, ello implica una perspectiva particular: nuestra mirada estará centrada en la PEA, por lo que analizaremos el vínculo entre estas naciones desde dicho marco. Para ello, partiremos de una delimitación conceptual de "política exterior" y utilizaremos la noción de "variable persistente" para analizar algunos aspectos que consideramos claves en la PEA en general y para este vínculo en particular. Entre ellos, le prestaremos especial atención a lo que denominaremos "latinoamericanismo". Así, buscaremos entender cómo algunos aspectos generales e históricos de la PEA se entrecruzan e inciden en la relación del país con México. El 


\section{LA INCIDENCIA DE LAS VARIABLES PERSISTENTES DE LA POLÍTICA EXTERIOR ARGENTINA: EL CASO DE LA RELACIÓN CON MÉXICO}

\section{MARÍA JULIA FRANCÉS}

objetivo del trabajo será analizar la incidencia de estas variables persistentes de la política exterior argentina sobre el caso de la relación de este país con México durante las administraciones de Cristina Fernández. A partir del análisis de una de estas variables, nos preguntaremos si, con sus conflictos y coincidencias, las características del vínculo a partir del accionar externo argentino se enmarcan en una verdadera estrategia latinoamericanista más allá de la subregión sudamericana y si los demás aspectos analizados de la PEA inciden positiva o negativamente sobre dicha cuestión. Para ello, nos centraremos en las dos administraciones de Cristina Fernández con especial atención a cuatro variables: el economicismo, el juridicismo que conjugaremos con la problemática de los recursos naturales y el territorio, para finalmente enfocarnos en el vínculo con la región y el concepto de latinoamericanismo.

\section{Consideraciones preliminares}

En primer lugar, es importante aclarar qué entendemos por política exterior. Con esta noción nos referimos a una serie de acciones, posiciones, estrategias y decisiones que lleva a cabo un Estado para vincularse con otros actores del sistema internacional. La consideramos una política pública fruto de un conjunto de interacciones que son el resultado de una serie de reglas, estructuras, valores y principios que conforman el régimen político de una sociedad ${ }^{\mathrm{II}}$. Compartimos la idea que se aleja del realismo más estricto para postular que se deben analizar de las variables internas para comprender las decisiones que un Estado toma al relacionarse con los demás. En este sentido, rescatamos los planteos de Lasagna, que considera que un régimen político está compuesto por las relaciones entre el Estado y la sociedad, una serie de principios y valores que determinan su orientación y un conjunto de factores estructurales.

Todo ello influye en tres dimensiones que conforman la política exterior: intereses, objetivos y estrategias, su proceso de elaboración y el estilo diplomático. Así, Lasagna (1996) afirma que las creencias básicas de un grupo gobernante sobre las cuestiones internacionales y su posicionamiento, por ejemplo, en el espectro ideológico político de izquierda-derecha pueden darle determinadas orientaciones. Estas consideraciones nos permiten ubicarnos en un contexto particular de las relaciones entre Argentina y México. Podemos afirmar que dos décadas antes del período bajo estudio, ambos países pasaron por un cambio de régimen que afectó tanto a sus modelos de desarrollo internos como a sus decisiones de política exterior y, por lo tanto, su actuación en el sistema internacional. Estos años precedentes estuvieron marcados por crisis económicas y políticas de gran envergadura para ambos países y consecuentes cambios en los objetivos y las prioridades de sus agendas externas. Ambos pasaron por etapas de implementación de distintos aspectos del denominado neoliberalismo, en particular una gran apertura económico-comercial, aunque con diferencias relevantes. En relación con ello, durante la década de los 90, firmaron los tratados de libre 


\section{LA INCIDENCIA DE LAS VARIABLES PERSISTENTES DE LA POLÍTICA EXTERIOR ARGENTINA: EL CASO DE LA RELACIÓN CON MÉXICO}

\section{MARÍA JULIA FRANCÉS}

comercio que se convertirían en elementos fundamentales de sus políticas exteriores: el Mercado Común del Sur y el Tratado de Libre Comercio de América del Norte (TLCAN). En ambos casos, en el marco de la globalización, las políticas exteriores se concentraron cada vez más en aspectos económicos y vieron a sus áreas de integración como bases para la vinculación con el resto de la región y con el mundo.

Más allá de los períodos precedentes, nos parece interesante destacar también para el caso bajo estudio que Lasagna considera significativos para el análisis los atributos subjetivos, incluso aquellos relacionados con la personalidad del liderazgo, que pueden determinar la forma de conducción de la política exterior, llamado por el autor "estilo diplomático"III. Esto nos permite afirmar que algunas características presidenciales pueden tener incidencia en la política exterior de los países bajo análisis $\mathrm{y}$, junto a la ideología, pueden generar una mayor o menor sintonía entre ellos. Teniendo en cuenta estas consideraciones, el presente trabajo busca realizar un análisis que ofrezca cierta continuidad en esta dimensión, de manera tal que podamos plantear generalizaciones sobre ciertos aspectos de la relación. En el caso bajo estudio, buscamos un recorte temporal que coincidiera con las administraciones de Cristina Fernández en Argentina y con los mandatos de dos presidentes mexicanos de distintos partidos pero con ciertas semejanzas entre sí (Felipe Calderón y Enrique Peña Nieto), superado el sexenio de Vicente Fox ${ }^{\mathrm{IV}}$. Tomamos en cuenta el liderazgo en México y sus estrategias de política exterior porque creemos que el accionar externo de nuestro país no solo responde a las características generales el sistema internacional sino, en el caso de una relación bilateral o regional, debe tener "reflejos" y evaluar los cambios producidos en las políticas exteriores de otro estado, en este caso México, como plantea Miranda (2004).

Lo anterior no implica que en este análisis se sostenga una postura determinista respecto a las variables de política interna sobre el accionar exterior de los Estados. Por el contrario, reconocemos que pueden tener distintos grados de incidencia según, por ejemplo, los límites y las condiciones que impongan las características del Sistema Internacional. Y es justamente esta una de las particularidades de la política pública bajo estudio que la distingue de otras y la constituye como objeto de un campo disciplinar diferenciado. Así, si concentramos la definición en el accionar externo del actor estatal, podemos plantear con Russell (1990) que es su proyección en el ámbito externo frente a una amplia gama de actores e instituciones gubernamentales y no gubernamentales tanto en el plano bilateral como multilateral y está conformada por tres dimensiones analíticamente separables: la político- diplomática, la estratégico-militar y la económico-comercial.

Ver a la política exterior como una proyección del accionar del Estado en el ámbito internacional pone de relieve las diversas dimensiones de los vínculos pero también incluye en su interior las relaciones que se dan en el marco de instituciones multilaterales, tanto de manera coyuntural como a largo plazo. En el trabajo realizado 


\section{LA INCIDENCIA DE LAS VARIABLES PERSISTENTES DE LA POLÍTICA EXTERIOR ARGENTINA: EL CASO DE LA RELACIÓN CON MÉXICO}

\section{MARÍA JULIA FRANCÉS}

por el OPEA, esta multidimensionalidad nos lleva a tener en cuenta aspectos tanto bilaterales como temas de la agenda externa.

El interés principal de este capítulo es el análisis de un caso particular delimitado geográfica y temporalmente para comprender observar las denominadas "variables persistentes" que la teoría encuentra a lo largo de la historia de la PEA. El estudio particular y acotado nos permite ver con mayor profundidad cómo se entrecruzan estas variables y cómo inciden en un vínculo bilateral concreto.

Con lo anterior nos referimos al esquema de la Dra. María del Pilar Bueno (2014), coordinadora del OPEA, sobre la continuidad y el cambio en la PEA a lo largo de la historia. Ubicándonos dentro de la teoría de la autonomía de Puig, la autora retoma sus planteos sobre las tendencias profundas de la PEA para luego añadir nuevos conceptos. Por un lado, para el autor el problema de la inserción se da en las distintas fases de la historia argentina e implica una tendencia autonómica o dependiente en las acciones de política exterior. Junto a ella, este identificaba la relación con Estados Unidos (y, como señala Bueno, su vínculo con el europeísmo), con América latina y la cuestión territorial/recursos naturales como factores que a lo largo del tiempo fueron interactuando y determinando el modelo de política exterior.

Asimismo, Bueno plantea que estos elementos pueden ir acompañados de otras constantes que tienen distintas características según de qué modelo se trate, es decir, nuevamente tendiente a la dependencia o a la autonomía. A partir de los planteos de Gustavo Ferrari (1981) y argumentos propios, la autora encuentra útil para su análisis añadir las siguientes categorías: el juridicismo (o escapismo por el derecho); y el economicismo $^{\mathrm{V}}$. Así, podemos pensar en un modelo para el análisis histórico y actual de la PEA a partir de la relación de estas llamadas "variables persistentes" y el entramado que se va conformando entre ellas en cada fase de la PEA. Esto no significa que la Argentina se relacione con el Sistema Internacional de una manera estable o constante, sino que estas categorías nos permiten una comprensión más profunda tanto de sus cambios como de sus continuidades para ver conjunto de las decisiones de política exterior tomadas por el país.

El análisis anterior no implica que planeemos una política exterior estable argentina a lo largo del tiempo y por ello reconocemos su volatilidad, así como la reciente espontaneidad en sus respuestas ante los cambios en el sistema internacional ${ }^{\mathrm{VI}}$. Si bien en el presente trabajo no es pertinente desarrollar cada una de estas categorías, consideramos algunas de ellas para el análisis al que nos abocaremos.

Con todo, nos será útil retomar la cuestión del economicismo como variable permanente, refiriéndonos a la búsqueda argentina de una inserción internacional basada en su modelo económico, ya desde la etapa fundacional agroexportadora ${ }^{\mathrm{VII}}$. También, a partir de la crisis de 2001, las limitaciones y los condicionantes a las relaciones internacionales argentinas que resultaron de su default y la influencia del modelo económico de las administraciones kirchneristas sobre los objetivos de PEA, se 


\section{LA INCIDENCIA DE LAS VARIABLES PERSISTENTES DE LA POLÍTICA EXTERIOR ARGENTINA: EL CASO DE LA RELACIÓN CON MÉXICO}

\section{MARÍA JULIA FRANCÉS}

vieron reflejados en el accionar internacional del país. Esto nos permite afirmar que el estudio de las relaciones económico-comerciales que lleva adelante la Argentina debe tener un lugar especial en todo análisis sobre la PEA.

Por otra parte, en cuanto al alto perfil en las relaciones con América Latina, la primera variable persistente antes enumerada, utilizaremos los planteos de otros autores que nos permiten llegar al cuestionamiento acerca de la dicotomía sudamericanismolatinoamericanismo. Desde una perspectiva retórica, encontramos históricamente tanto referencias al sentimiento de pertenencia de Argentina respecto a América Latina como consideraciones que desdeñan el vínculo con la región en su conjunto y diferencian al país de su entorno geográfico para acercarlo a otras regiones, en particular, la europea. Esta oscilación puede evidenciarse en las acciones de la nación desde sus inicios, tanto en su participación en foros que reivindicaban el panamericanismo como en la reafirmación de su condición de "excepcionalidad" frente a sus vecinos.

En relación con lo anterior, vemos que las variables vínculo con Estados Unidos/Europa y la jerarquización de América latina inciden de distintas maneras según la fase histórica analizada e influyen una sobre la otra. En este sentido, consideramos especialmente revelador el análisis realizado por Pablo Yankelevich (2000) en cuanto a la trayectoria del vínculo entre México y Argentina. El autor plantea que a principios del siglo XX, dados ciertos espacios de "autonomía" logrados por la Argentina a través de su vínculo con Europa que le permitían cierto discenso con los Estados Unidos y la autodefinición argentina como un poder que podía ponerle límites en la región, México buscaba, tras su revolución de 1910, la aprobación del país sudamericano y para ello intentó fomentar una política argentina favorable tanto para desarrollar la relación bilateral como para tener su apoyo en foros multilaterales ${ }^{\mathrm{VIII}}$. Así, vemos que las variables influyen entre sí y son separables solo de manera teórica y analítica.

Más adelante, y en particular entrado del siglo XX, podemos encontrar análisis que vislumbran elementos latinoamericanistas (con sus distintas definiciones) en la PEA, en muchos casos superando la perspectiva subregional (sudamericana). En relación con ello, autores como Miranda (2010) consideran que el latinoamericanismo es un posicionamiento histórico de la PEA (si bien intercalado con visiones occidentalistas), al tiempo que afirman que esta relación es un tema "insoslayable". Esto se debe a una serie de nociones que se ponen en juego, como la idea de pertenencia, el concepto de vecindad y las consideraciones geoeconómicas. En este sentido, Miranda considera que la PEA jerarquiza a América Latina, en particular a partir del retorno a la democracia, en el momento en que el país asume junto a sus socios continentales "la defensa de la región, la democracia y la solidaridad diplomática"IX

El mismo autor, sin embargo, plantea que fueron cambiando la densidad y la jerarquía del vínculo con la región en la agenda externa argentina según la administración que la llevara adelante. En este sentido, nuevamente inciden las otras variables persistentes para configurar el modelo de PEA llevado adelante. Con la 


\section{LA INCIDENCIA DE LAS VARIABLES PERSISTENTES DE LA POLÍTICA EXTERIOR ARGENTINA: EL CASO DE LA RELACIÓN CON MÉXICO}

\section{MARÍA JULIA FRANCÉS}

recuperación de la democracia, surgieron además nuevas cuestiones de agenda, como la de derechos humanos y la deuda externa, que la acercaron a la región, dadas las problemáticas comunes. El economicismo, sin embargo, fue haciendo cada vez más pronunciado el relacionamiento argentino a través de los vínculos comerciales y financieros, expresión de ello es en particular la relevancia asignada a la negociación entre México y el Mercosur llevada adelante en la época.

Pero nuevamente destacamos la importancia de los factores internos, no solo del cambio de régimen político sino de los límites y desafíos que impone. Así, la PEA culminó por concentrarse únicamente en Sudamérica luego de la crisis de $2001^{\mathrm{X}}$ como una estrategia de "refugio" luego de su colapso multidimensional. El alto perfil que encuentra Miranda en la relación con esta última coincide con lo que algunos autores consideran una variable persistente y otros, una ruptura con tradiciones anteriores. Según Miranda, la nueva jerarquía responde a la apuesta argentina poscrisis por un posicionamiento que le permitiera satisfacer sus intereses. Podríamos también considerarlo la aplicación de una estrategia nueva en otro intento de "reinserción en el Sistema Internacional".

En este punto es relevante preguntarnos por qué definición de latinoamericanismo optaremos en el presente trabajo y de qué manera se diferenciaría de una perspectiva sudamericanista. En primer lugar, utilizaremos el concepto de una manera amplia y laxa. Para afirmar que una PE es latinoamericanista, buscaremos comprobar si existe una estrategia de política exterior de vinculación sistemática y multidimensional con los demás países latinoamericanos, tanto de manera bilateral como en ámbitos multilaterales, más allá de acciones específicas y coyunturales. Definimos estrategias como el conjunto de objetivos o metas e instrumentos seleccionados entre un rango de posibilidades para promover determinados intereses ${ }^{\mathrm{XI}}$. En este sentido, cabe preguntarnos por el mayor o menor apoyo a la construcción de instituciones y mecanismos de diálogo (bi y multilaterales) que superen el corto plazo o el plano discursivo de manera que plasmen en actos concretos los valores y principios esgrimidos retóricamente.

Con fluctuaciones, según autores como Miranda (2010), dicha definición es aplicable a la vinculación de Argentina con Sudamérica y en particular sus países limítrofes, pero no ya a toda América Latina. El autor plantea que existe un eje cercanía-lejanía que da cuenta de la jerarquía de las distintas naciones latinoamericanas en la agenda argentina. Esta escala permite describir el cambio producido en la PEA actual respecto a administraciones anteriores. Así, con vaivenes coyunturales, Argentina mantuvo una intensa asociación con sus vecinos fronterizos y en particular con Brasil, a pesar de los obstáculos que encontró el principal mecanismo de integración de la subregión: el Mercado Común del Sur. Miranda considera que un ejemplo importante es el caso de Chile, pero también remarca el rol de Venezuela ${ }^{\mathrm{XII}}$. 


\section{LA INCIDENCIA DE LAS VARIABLES PERSISTENTES DE LA POLÍTICA EXTERIOR ARGENTINA: EL CASO DE LA RELACIÓN CON MÉXICO}

\section{MARÍA JULIA FRANCÉS}

$\mathrm{Si}$ añadimos a las consideraciones anteriores una visión que tome en cuenta no solo el aspecto geográfico, sino también las distinciones políticas, económicas e históricas entre las subregiones latinoamericanas, podemos hablar de un escenario latinoamericano del Norte y otro del Sur ${ }^{\mathrm{XIII}}$, que puede coincidir con el eje mencionado anteriormente. Así, los autores vislumbran dos potencias medias en dichas subregiones: México y Brasil. Estas ejercen su liderazgo e interactúan con la potencia hegemónica (Estados Unidos) de distintas maneras. Este planteo le da particular relevancia al vínculo entre Argentina y México y añade otro aspecto interesante al análisis.

Nos resultan reveladores en este sentido algunos de los planteos de México durante el proceso de negociaciones para la firma de un acuerdo con el Mercosur. Vemos que sus funcionarios en ese contexto identificaban la inserción de México en el mundo a partir de su relación con América del Norte y en particular a través de su tratado de libre comercio, el TLCAN. Pero consideraban que su país podía funcionar como puente entre las potencias del norte y las economías emergentes del sur ${ }^{\mathrm{XIV}}$. Más adelante, desestimado el proyecto de conformación de un área de libre comercio en toda América, México volvió a priorizar sus relaciones "naturales" con Centroamérica, zona que, a su vez, Estados Unidos considera su "patio trasero". De esta manera, el vínculo entre la Argentina y México puede analizarse desde la perspectiva de su interacción con los otros actores subregionales, teniendo en cuenta el triángulo Argentina-BrasilMéxico y Argentina-Estados Unidos-México. Esto sin olvidar las posibles instancias de rivalidad que se fueron configurando en años recientes entre los dos líderes subregionales, México y Brasil ${ }^{\mathrm{XV}}$.

En el mismo orden de ideas, Miranda (2011) considera que la función de México es la de "nexo" entre América del Norte y Centroamérica, para obtener beneficios gracias a su vínculo especial con Estados Unidos. La relación de este último actor con Argentina no es tema del presente trabajo, pero las consideraciones anteriores implican también que dicha república sudamericana deberá formular respuestas a su posible marginación de la agenda mexicana, toda vez que, como mencionamos más arriba, la relación argentino-estadounidense tiene históricamente momentos de tensión ${ }^{\mathrm{XVI}}$.

En cuanto al segundo actor relevante, Centroamérica, como fue analizado en estudios anteriores ${ }^{\mathrm{XVII}}$, vemos que tiene importancia esporádica y un papel errático en la PEA, aunque no siempre desdeñable. Si bien no podemos concluir que exista una estrategia sistemática y a largo plazo con dicha subregión, sí observamos presencia argentina en áreas de relevancia para la política mexicana. Esto se evidencia en casos como los proyectos de cooperación técnica, por ejemplo. Asimismo, cabe destacar un flujo de comercio continuado y ampliamente beneficioso para la Argentina, aunque este tuvo distintos niveles de relevancia en las relaciones con cada una de dichas naciones. De esta manera, la PEA no entró en conflictos sustanciales con México en lo que Miranda denomina su "esfera de influencia", ni tuvo una política global y a largo plazo 


\section{LA INCIDENCIA DE LAS VARIABLES PERSISTENTES DE LA POLÍTICA EXTERIOR ARGENTINA: EL CASO DE LA RELACIÓN CON MÉXICO}

\section{MARÍA JULIA FRANCÉS}

respecto a ella, pero la Argentina tampoco estuvo ausente de esta área geográfica más "alejada" según el eje cercanía-lejanía mencionado anteriormente.

La distinción propuesta entre dos escenarios que coinciden con una separación geográfica concreta puede resignificarse también al plantear la importancia de la dimensión interna que nos lleva a revisar las diferencias ideológicas tanto en sus regímenes políticos en general como entre sus líderes. Esto nos permite explicar algunas de las relaciones más fluidas entre vecinos. Como afirmamos al comienzo de esta sección, consideramos que para la comprensión de la política exterior de un actor estatal es relevante considerar el aspecto doméstico. Ya mencionamos que la crisis de 2001 puede explicar al menos una parte el cambio que algunos autores ven en la tradición de la PEA. Miranda (2010), por su parte, plantea la importancia del vínculo personal entre los jefes de Estado y resalta las rispideces existentes en el caso particular de Argentina y México con el presidente Vicente Fox. En relación con ello, reconocemos las dificultades que puede implicar la acotada selección temporal del presente estudio que no nos permite rastrear constantes a lo largo de las distintas administraciones de ambos países. Sin embargo, buscaremos enfocarnos en aspectos que superen las consideraciones cortoplacistas.

A partir de nuestra definición de latinoamericanismo y de las consideraciones precedentes, buscamos elementos concretos que relativicen la "lejanía" geográfica y la distancia entre las dos subregiones latinoamericanas mencionadas anteriormente.

Como último aspecto a tener en cuenta, recordamos que México y Argentina, el firmaron en 2007 un Acuerdo de Asociación Estratégica (AAE), cuyas instituciones y mecanismos de concertación se afianzaron a partir de la segunda reunión de las comisiones mixtas y la firma del reglamento interno del Consejo de Asociación Estratégica en 2011. Así, otra razón para la selección del marco temporal para el presente análisis es el interés por el que fue anunciado como un nuevo período en los vínculos entre estos dos países a partir del AAE.

\section{Economicismo}

A principios del siglo XX, las relaciones entre Argentina y México eran escasas. Con la Revolución de 1910, muchos países del continente se habían alejado de su socio azteca y este se encontraba con importantes dificultades económicas, políticas y sociales. Yankelevich plantea que la política exterior mexicana buscó estrechar vínculos incluso con un país tan alejado como Argentina a través de una diplomacia "intelectual", de las letras y el arte, para cambiar la imagen México en el mundo y, para ello eligió la dimensión económico-comercial, pensando que podría ser una cuestión atractiva para la Argentina y que incentivaría su acercamiento. Así, la dimensión económica de la PE mexicana se supeditó en realidad a objetivos políticos. 


\section{LA INCIDENCIA DE LAS VARIABLES PERSISTENTES DE LA POLÍTICA EXTERIOR ARGENTINA: EL CASO DE LA RELACIÓN CON MÉXICO}

\section{MARÍA JULIA FRANCÉS}

A finales del mismo siglo, fue nuevamente la política exterior mexicana la que buscó un acercamiento con su socio conosureano a través del comercio, como uno de los bastiones para la firma de un acuerdo con el Mercosur. La Argentina, por su parte, también se enfocó en este aspecto de la relación y sus funcionarios se congratularon por las consecuencias positivas que podría tener un tratado de libre comercio entre ambos para el vínculo en general. Sin embargo, estas negociaciones fracasaron, al igual que las de principios de siglo, y la excesiva concentración de los esfuerzos en este aspecto tensó las relaciones bilaterales.

Al acercarnos al período actual, como planea Bueno (2014), la política comercial fue uno de los pilares de la política del kirchnerismo, que llevó a sus administraciones a buscar ampliar los destinos de exportación y la oferta exportable. Entendemos aquí por economicismo "el condicionamiento de la política económica sobre la política exterior en sus distintas dimensiones", con la consecuencia de que en la última década la "política exterior fue pormenorizada y subordinada a la política económica. Esto conllevó que la prioridad en la inserción argentina en el mundo, estuvo dada por la materia económica-comercial como resulta del discurso de Kirchner". Asimismo, dentro de la dimensión económica, encontramos una predominancia del interés por el comercio, a tal punto que Bueno (2014) afirma que "la política de inversiones no ha tenido siquiera comparación con la relevancia" de aquella.

Para el período bajo estudio, es natural suponer que una concentración en la dimensión económico-comercial de la relación pudo también generar más desencuentros que acercamientos debido a la "poca sintonía" entre ellos. México y Argentina sostienen valores y principios diferentes respecto al comercio internacional. Mientras que las dos administraciones mexicanas durante este tiempo se plantearon como objetivo el fomento del librecambio y mantuvieron su tratado de libre comercio con Estados Unidos como determinante para su inserción internacional ${ }^{\mathrm{XVIII}}$, Argentina tomó medidas que muchos consideraron proteccionistas y encontró grandes obstáculos para profundizar su propio proceso de integración.

Las tensiones en la relación provocadas por las diferencias en las perspectivas económicas venían ya incidiendo en el vínculo, en particular durante la administración de Vicente Fox. El rechazo de la Argentina a la constitución de un área de libre comercio en todo el continente durante la cumbre de Mar del Plata había llevado a una interacción conflictiva entre el entonces presidente Néstor Kirchner y su par mexicano que enfrió los lazos bilaterales en especial en su dimensión política, al menos hasta la llegada del presidente Calderón.

Llegado este punto, consideramos pertinente dar un panorama general de la dimensión económica del vínculo bilateral durante el período estudiado. Primero, debemos señalar que el AAE bilateral contó con una evidente impronta economicista, tal que de los cinco objetivos enumerados en su introducción, tres se relacionaron estrechamente con aspectos económico-comerciales. Segundo, Argentina y México con 


\section{LA INCIDENCIA DE LAS VARIABLES PERSISTENTES DE LA POLÍTICA EXTERIOR ARGENTINA: EL CASO DE LA RELACIÓN CON MÉXICO}

\section{MARÍA JULIA FRANCÉS}

importantes cuotas de inversión extranjera directa (IED) entre sí. Las empresas argentinas como Techint tienen una participación significativa en la industria metalúrgica mexicana desde hace tiempo, mientras que en el período bajo estudio, cobraron importancia nuevos aportes a dicho rubro ${ }^{\mathrm{XIX}}$. Argentina representa uno de los principales inversionistas sudamericanos en el país, si bien registró una significativa desinversión que rompió con la tendencia al alza de años anteriores. Mientras tanto, México aportó importantes fondos para el sector de las telecomunicaciones y el turismo en la Argentina, entre otras áreas ${ }^{\mathrm{xx}}$.

Respecto al comercio, cabe aclarar que, hasta el año 2011, estas relaciones bilaterales se regían por dos Acuerdos de Complementación Económica (ACE 6 y 55) firmados en el marco de dos mecanismos de integración regional: la ALADI (1987) y el Mercosur (2002). Desde la firma del segundo, el flujo comercial bilateral fue incrementando a un ritmo superior al de otros socios y se llegó a liberalizar el intercambio de vehículos ligeros para el año 2006. Hasta el período 2007-2009, el comercio venía creciendo a una tasa promedio del $36 \%$. Si bien la crisis internacional de 2008-2009 significó una importante caída tanto de importaciones como de exportaciones entre ambos países, entre 2010 y 2011 dichas actividades lograron nuevamente un aumento significativo. La balanza comercial mostraba un crecimiento acumulado entre 2002 y 2001 de un 272\% (CCMA, 2012). Un dato interesante es que más del $90 \%$ de esta interacción estuvo constituida por la venta de bienes industriales (97\% en el caso mexicano, $85 \%$ en el caso argentino) principalmente del rubro automotor $^{\mathrm{XXI}}$.

Luego de la crisis internacional, la balanza comercial entre ambos, que venía presentando hasta 2010 un superávit promedio de 483 millones de dólares a favor de la Argentina, vio una reversión en su tendencia, de manera tal que México logró una diferencia a su favor de casi 676 millones de dólares que continuó a lo largo del período que analizamos (CCMA, 2012).

Lo anterior fue visto como un aspecto problemático en la relación por parte de la Argentina, que necesitaba una balanza comercial superavitaria para su modelo económico. Como planteábamos al comienzo del parágrafo, las administraciones kirchneristas buscaron resultados más favorables para el país en el intercambio internacional. El caso de México es notable ya que se trata de una compra y venta de bienes altamente industrializados, es decir, un perfil diferente al de sus balanzas deficitarias con países desarrollados, y con un socio emergente con importancia creciente (al menos hasta 2009) para su comercio. La relevancia de la búsqueda de reducción del déficit por parte de las políticas desarrolladas por Argentina desembocó en una decisión que tensó las relaciones bilaterales. En 2012 Argentina suspendió las ventajas otorgadas a su socio a través del Mercosur en el marco del ACE 55. Luego de que Brasil negociara una revisión de dicho tratado y dado el creciente déficit comercial en la balanza mexicanoargentina, la administración de Cristina Fernández había 


\section{LA INCIDENCIA DE LAS VARIABLES PERSISTENTES DE LA POLÍTICA EXTERIOR ARGENTINA: EL CASO DE LA RELACIÓN CON MÉXICO}

\section{MARÍA JULIA FRANCÉS}

solicitado sin éxito la renegociación del acuerdo. El resultado fue una controversia con el país norteamericano que las instancias institucionalizadas de diálogo no lograron resolver con la celeridad necesaria. La situación finalmente llevó a que México se uniera a una serie de países que presentó reclamos ante la OMC contra las políticas comerciales argentinas, llamando a la conformación de un panel de solución de diferencias.

En el conflicto se puso en juego otro aspecto económico: el temor de la Argentina de que un nuevo acuerdo entre Brasil y México generara una desviación de comercio en detrimento de su economía. La inquietaba que el tratado entre ambas potencias subregionales fomentara la producción únicamente entre ellas, lo cual

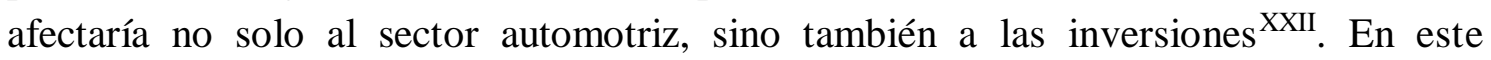
conflicto vemos también que el discurso oficialista buscó reivindicar sus decisiones de política comercial, en particular luego de las quejas ante la OMC. Pero su argumentación intentaba sostener que solo las grandes potencias estaban en desacuerdo, impulsadas por EE.UU., y que los países latinos no habían sido perjudicados. Sin embargo, la participación mexicana al respecto fue difícil de soslayar y podemos suponer que esto pudo incidir en el interés de la Argentina por buscar una pronta resolución. De esta manera, el caso de México fue presentado como una situación particular de más fácil salida y de inmediato se buscó el diálogo con dicho país para llegar a una negociación. Tres meses más tarde, con la firma de un nuevo acuerdo, se restringió el libre comercio existente en el sector automotriz y se impuso una serie de cupos anuales para estas mercancías (ICTSD, 2012).

Argentina logró una salida de este conflicto al impulsar una gira de la ministra de Industria argentina, Débora Giorgi, y el nuevo documento firmado la llevó a anunciar otro "relanzamiento" de la relación bilateral"XXIII que, según los funcionarios argentinos, sería coadyuvado por el cambio de administración en México. Así, los medios oficialistas argentinos sugirieron que las diferencias como esta se debían más al alineamiento de México con EE.UU. que a un distanciamiento entre latinoamericanos. En este sentido, retomaron una temática recurrente en la PEA en relación a la tensión con dicha potencia mundial para explicar la particular situación acaecida y sostuvieron que un gobierno mexicano menos alineado con ella podría facilitar las relaciones argentino-mexicanas (Página 12, 14/12/2012). Sin embargo, estos hechos nos permiten ver cómo la Argentina llevó adelante una política "poco amistosa" con México por cuestiones comerciales, arriesgándose a tensar la relación bilateral incluso en el marco de una "relación estratégica".

Otro ejemplo de la importancia de la dimensión comercial para el vínculo entre Argentina y México es que algunos de los encuentros más importantes entre ministros de ambos países se dieron en el marco de misiones comerciales argentinas hacia su socio norteamericano. Este es el caso de los encuentros durante 2013, algunas de las reuniones de las comisiones del AAE, etc. Así, el interés argentino por mejorar su 


\section{LA INCIDENCIA DE LAS VARIABLES PERSISTENTES DE LA POLÍTICA EXTERIOR ARGENTINA: EL CASO DE LA RELACIÓN CON MÉXICO}

\section{MARÍA JULIA FRANCÉS}

balanza comercial con México no se restringió a la imposición de cuotas para el sector automovilístico. Su relevancia puede verse con mayor claridad en el anuncio en 2014 del Programa de Aumentos y Diversificación de Exportaciones. En él, México aparece como uno de los quince países emergentes dinámicos considerados prioritarios, entre los cuales se encuentra solo un país latinoamericano más: Brasil. Es interesante destacar que el programa se plantea dos componentes con metas diferenciadas. Uno es el mencionado listado de países de mayor relevancia, con los que se buscará una mejora cuantitativa de las exportaciones. El otro es Latinoamérica con los objetivos de mejoras cualitativas e integración productiva, con particular énfasis en el sector industrial, más desarrollado en el caso mexicano (Presidencia de la Nación, 2014).

Otro aspecto económico relevante, que vincula el comercio con la inversión se da nuevamente en el sector automotor. La compañía General Motors presentó a finales del período analizado un plan denominado Fénix para la producción de vehículos en la Argentina. Este fue apoyado por la administración Fernández, que a su vez anunció que buscaría negociar con Brasil un acuerdo para el fomento de la integración productiva con estímulos para la fabricación de autopartes y también la integración junto a México una plataforma de producción y desarrollo de la industria (Conferencia de Jefe de Gabinete, 2014). ${ }^{\text {XXIV }}$.

Sobre la cuestión de las inversiones, cabe destacar que algunos académicos y financistas plantearon que la decisión política de la Argentina de nacionalizar de Yacimientos Petrolíferos Fiscales (YPF) podría ser un factor que desalentó a los aportes de capital mexicano en Argentina al generar rispideces en el aspecto económico entre ambos países, debido a la participación accionaria que tenía la petrolera mexicana PEMEX en Repsol, antiguo conductor de la firma. A pesar de ello, en una segunda etapa del conflicto, la Argentina buscó la negociación con México y su apoyo. La administración Fernández llegó a un preacuerdo con la empresa expropiada a fines de 2013 y se ocupó de recalcar "el preponderante rol que asumió el director general de PEMEX, Emilio Lozoya", quien por su parte incentivó al Consejo de Administración de Repsol para aceptar la propuesta argentina (América Economía, 2013). Asimismo, el funcionario argentino Carlos Zannini mencionó más tarde el apoyo del presidente Peña Nieto (así como de ministros españoles) e indicó que este fue clave para lograr el acuerdo (MercoPress, 2014).

Podemos concluir este parágrafo diciendo que en general, las administraciones kirchneristas no dudaron en tomar decisiones de política exterior que pudieran erosionar su vínculo con México en los casos en que consideraron que la cuestión era relevante para su modelo económico. Vemos así que lo que se percibió como conveniencia en estos aspectos pudo generar tensiones con México, que la Argentina resolvió con respuestas ad hoc que indicaron cierta "sobrevaloración de la espontaneidad", en términos de Bueno (2014). Asimismo, la dimensión económico- comercial estuvo en la palestra durante este período y determinó vaivenes en la relación. 


\section{LA INCIDENCIA DE LAS VARIABLES PERSISTENTES DE LA POLÍTICA EXTERIOR ARGENTINA: EL CASO DE LA RELACIÓN CON MÉXICO}

\section{MARÍA JULIA FRANCÉS}

\section{El juridicismo y los recursos naturales / cuestión territorial}

Argentina usa las herramientas del derecho internacional y el derecho domestico para legitimar sus decisiones en ambos planos. una disputa (politicoambiental como en el caso de uruguay) se vuelve un debate netamente juridico acerca del incumplimiento de un tratado internacional en menoscabo de las preocupacioens de los principales actores

La referencia a la relación bilateral del principios del siglo XX nos sirve también para introducir esta sección. Como afirma Bueno (2014), uno de los ejemplos más característicos del juridicismo de la PEA es la defensa de la fórmula de arbitraje y la doctrina Drago. Fue justamente esta característica de la PEA, en confrontación con la posición de Estados Unidos en el continente, la que atrajo, según Yankelevich, a la diplomacia mexicana en un intento por buscar un aliado contra su vecino del norte, aunque a su pesar no haya logrado una política argentina vigorosa en su defensa.

Para el período bajo estudio, la tendencia juridicista de la PEA tuvo consecuencias ambivalentes para el vínculo con México. Por un lado, podemos relacionarla con el enfrentamiento por la denuncia del ACE 55 y la consecuente presentación en contra de la Argentina presentada por México ante la OMC.

En este caso, si bien la negociación bilateral no se llevó a cabo en el marco institucional del arreglo de diferencias de la OMC ni del Mercosur, la Argentina buscó presentar la cuestión del diferendo como un problema de fácil resolución a través de la implementación de nuevos instrumentos jurídicos, sin debatir el problema de fondo que parecía haber sido planteado por México: la cuestión más general del proteccionismo llevado adelante por la administración. El planteo más general de la problemática por parte de México se vislumbró a través de su decisión de presentar un escrito en conjunto con otros socios comerciales de la Argentina que se quejaban de otros aspectos proteccionistas. Pero Argentina se esforzó por deslindar el caso mexicano del planteado por los países desarrollados y buscó una solución bilateral, eludiendo la negociación con los otros firmantes.

La sobresimplificación de la temática por parte Argentina se evidencia además en la falta de referencia a la cuestión más profunda acerca del accionar unilateral de Brasil en cuestiones comerciales. En este sentido, que el debate se restringiera a instrumentos jurídicos pasó por alto problemáticas más profundas que estaban en el origen del conflicto, pero permitió una resolución más expeditiva y pragmática de la cuestión, que de otro modo podría haber herido más profundamente la relación bilateral.

Analicemos ahora un caso se conjugan la variable "juridicismo" con la cuestión de los recursos naturales, donde se ve también la importancia de la dimensión económica en el vínculo bilateral, así como el uso discursivo de referencias a la pertenencia latinoamericana. es en la problemática surgida a partir de la nacionalización del 51\% de las acciones de la empresa Yacimientos Petrolíferos Fiscales (YPF) en 


\section{LA INCIDENCIA DE LAS VARIABLES PERSISTENTES DE LA POLÍTICA EXTERIOR ARGENTINA: EL CASO DE LA RELACIÓN CON MÉXICO}

\section{MARÍA JULIA FRANCÉS}

manos de la española Repsol. El caso es relevante para la relación entre México y Argentina, debido a la participación de la petrolera estatal PEMEX como accionista en Repsol. Si bien tanto Argentina como México tuvieron actitudes cambiantes a medida que se desarrolló el conflicto, la primera buscó legitimar su accionar a través del derecho interno y reducir la cuestión a las atribuciones de un estado respecto a sus recursos naturales.

Para comprender este tema, debemos recordar que el gobierno de Cristina Fernández expropió en abril de 2012 el 51\% de las acciones de Yacimientos Petrolíferos Fiscales (YPF) que eran propiedad de Repsol.

La administración kirchnerista argumentó, entre otras cosas, que existía una inversión deficitaria por parte de la firma española, que entre 2000 y 2010 había tenido una tasa de reparto de dividendos mayor al 100\%, lo cual implicaba una colocación de ganancias en otros proyectos. Además, consideró que el país debía controlar sus propias reservas energéticas de esta índole, como los demás países latinoamericanos ${ }^{\mathrm{XXV}}$. Mientras tanto, la empresa de capital mexicano PEMEX había incrementado su participación en la firma española y había buscado aumentar su control en el proceso de toma de decisiones interno, lo cual llevó a un enfrentamiento con sus directivos y malestar con el gobierno español. En este contexto, la decisión argentina respecto a sus recursos naturales era especialmente peligrosa para la política energética mexicana y su relación con Repsol.

El rápido proceso interno de aprobación de la medida en Argentina y los argumentos esgrimidos por esta, enfrentaron en un primer momento con un rechazo rotundo por parte del funcionarios mexicanos, en particular del presidente Calderón, que tildó la expropiación de poco "responsable y racional" y se consideró que esto dañaría la confiabilidad y previsibilidad en Argentina. Para México, la legitimidad de la medida no podía apoyarse entonces en el procedimiento interno argentino o en fundamentos de derecho sobre los recursos naturales o en referencias a las situaciones de los demás países latinoamericanos. La variable persistente relacionada a los recursos naturales incidió, entonces, en un principio, de manera negativa sobre la relación.

Es interesante notar que en este caso fue Repsol la que buscó herramientas jurídicas para reforzar su posicionamiento y amenazó con demandar (y efectivamente lo hizo) a aquellas empresas que llegaran a acuerdos con la "nueva" YPF para la explotación de yacimientos como el de Vaca Muerta, que la firma consideraba ilegítima. Pero nuevamente, más allá de las cuestiones legales, entra en juego la importancia de los recursos naturales y la cuestión energética y Argentina busca negociar con su socio norteamericano no solo la salida del diferendo con Repsol sino también la inversión en un proyecto que tiene para ella fundamental importancia: shale oil en Neuquén. De esta manera, los dos países cambiaron su posicionamiento frente a la cuestión y Argentina logró el apoyo mexicano y de PEMEX para llegar a un acuerdo negociado con la petrolera española. 


\section{LA INCIDENCIA DE LAS VARIABLES PERSISTENTES DE LA POLÍTICA EXTERIOR ARGENTINA: EL CASO DE LA RELACIÓN CON MÉXICO}

\section{MARÍA JULIA FRANCÉS}

El interés mexicano por los ofrecimientos argentinos lo llevó a criticar el accionar jurídico de Repsol contra empresas asociadas a YPF, como Chevron, e incluso a manifestar su apoyo al gobierno argentino por una de las cuestiones de fondo que el país intentó poner en juego desde un principio: el derecho de un estado para nacionalizar y controlar los recursos naturales. "Mal haríamos los miembros de una empresa que surgió de una nacionalización, cuestionar esas facultades al Estado argentino", afirmó Fluvio Ruiz Alarcón, asesor y miembro del Consejo Corporativo de Pemex, durante las negociaciones que realizó invitado por Buenos Aires para la explotación del reservorio de petróleo y gas no convencional. ${ }^{\mathrm{XVI}}$

Vemos así que la cuestión de los recursos jugó un papel importante en la relación bilateral tanto desde el punto de vista argentino como desde el mexicano, tanto por cuestiones internas como por su interés internacional.

La variable recursos naturales/ cuestión territorial vuelve a conjugarse con el juridicismo en el caso de la cuestión Malvinas. Bueno (2014) plantea que, con la excepción del enfrentamiento armado en 1982, este es otro caso de juridicismo en la PEA. En particular durante las administraciones de Cristina Fernández, este tema se vincula con los aspectos económicos y los recursos naturales, como los temas relacionados con la pesca y los hidrocarburos. Además, en este período, Argentina buscó asociar la cuestión con la denominada "militarización del Atlántico Sur". La política exterior argentina respecto a las distintas aristas de la temática se llevó adelante en distintos frentes. En este apartado nos interesa mencionar en particular la búsqueda persistente de muestras que refrenden el apoyo de aquellos países que tradicionalmente han acompañado los reclamos argentinos. Este es el caso de México, cuya posición favorable a los intereses de su socio fue mencionada y repetida por la diplomacia argentina en la gran mayoría de los comunicados conjuntos producidos por sus cancilleres y ministros, así como por los funcionarios del país en ámbitos regionales y multilaterales. En los informes de prensa emitidos durante el período tras este tipo de reuniones, observamos que aparecía la cuestión Malvinas como un tema de la agenda, aunque solo fuera para reiterar el apoyo mexicano. Así, la estrategia de la administración de Fernández tendió a incluir el tema en todo foro internacional donde fuera posible, enfatizando siempre el respaldo logrado de parte de otros países, como México.

Además de los foros multilaterales, Argentina tuvo durante este período una fuerte política de apoyo a la constitución de ámbitos de debate sobre la cuestión en distintos países. Un ejemplo de ello fue su incentivo para la constitución en 2011 del Grupo Mexicano de Solidaridad con las Islas Malvinas, relevante por ser uno de los primeros de su tipo. Asimismo, la diplomacia argentina incentivó a través de su embajada a la convocatoria por parte de la Universidad Autónoma de México convocaron al I Encuentro de Grupos de Solidaridad con la Cuestión Malvinas de América del Norte. México funcionó como un posible impulsor del diálogo con 


\section{LA INCIDENCIA DE LAS VARIABLES PERSISTENTES DE LA POLÍTICA EXTERIOR ARGENTINA: EL CASO DE LA RELACIÓN CON MÉXICO}

\section{MARÍA JULIA FRANCÉS}

académicos y participantes de Canadá y Estados Unidos, países que, por su histórica relación con Gran Bretaña, se encuentran más alejados de la posición Argentina. El resultado del diálogo fue la Declaración de México "en la que recordaron la subsistencia de la controversia de soberanía entre la Argentina y el Reino Unido, destacaron el mandato constitucional argentino de resolver esa disputa de manera pacífica, respetando los intereses de los habitantes de las islas y alentaron a la parte británica a restablecer el diálogo, de conformidad con las resoluciones de Naciones Unidas " (Embajada Argentina, 19 de junio de 2013).

\section{Vínculo con América Latina}

La cuestión Malvinas nos remite a la última variable persistente que trataremos, el vínculo con América Latina, ya que las consideraciones anteriores respecto a la incidencia de la cuestión en la relación bilateral son más o menos aplicables a los demás países latinoamericanos y la cuestión ha generado un discurso por parte de Argentina que hace constante referencia a que la temática incumbe a toda la región. Así, vemos que este vínculo entra en un juego complejo con otras variables persistentes. Bueno (2014) al respecto plantea que para entender sus connotaciones en las distintas etapas de la PEA, es necesario tener en cuenta el binomio Estados Unidos - Europa. Esta consideración cobra aun mayor importancia para nuestro estudio, al concentrarnos en la PEA hacia un país fuertemente marcado por su interacción con Estados Unidos y que, como afirma González González (2005) "mira de reojo" a su socio del Norte al definir su propio posicionamiento ante la región.

En este punto, conviene hacer algunas aclaraciones. En primer lugar, como hemos visto, el vínculo con América Latina fue cambiando a lo largo de la historia de la PEA y plantearlo como variable persistente no implica que consideremos que esta tuvo una estrategia continuada y coherente hacia la región. Tampoco significa que entendamos que México fue priorizado en la agenda de la PEA por tratarse de un país latinoamericano. Lo que buscamos en este apartado es entender de qué manera incidieron en la política argentina hacia México las características de su vinculación con la región en su conjunto y con los países que la constituyen. Esto nos remite al concepto de latinoamericanismo que planteábamos en la introducción y nos lleva a pensar en sus distintas interpretaciones.

Algunos autores utilizan el término para hacer referencia simplemente a la interacción con una o varias naciones de la región por fuera del vínculo con Estados Unidos o los socios europeos tradicionales. En este sentido, la relación "especial" con Brasil y sus vecinos limítrofes puede ser "latinoamericanismo", sin necesidad de una estrategia general para el conjunto de la región o una jerarquización de las relaciones bilaterales con todos sus socios regionales. Desde esta perspectiva, instancias de acercamiento concretas de la argentina hacia México como su Acuerdo de Asociación 


\section{LA INCIDENCIA DE LAS VARIABLES PERSISTENTES DE LA POLÍTICA EXTERIOR ARGENTINA: EL CASO DE LA RELACIÓN CON MÉXICO}

\section{MARÍA JULIA FRANCÉS}

Estratégica o el incremento de su relación comercial demostrarían el latinoamericanismo de la PEA. Sin embargo, como indicábamos al principio, consideramos que este concepto supera las acciones esporádicas o los acercamientos individuales.

Una segunda idea posible de latinoamericanismo está asociada a la apuesta argentina por la integración subregional en el marco del Mercosur o la participación en mecanismos como la Unasur. En este caso vemos que se confunde América Latina con Sudamérica y, ante la clara importancia que cobró en particular el Cono Sur durante las administraciones bajo estudio, desdeña el rol de otros actores regionales para la PEA. Esta visión no toma en cuenta las diferenciaciones que realizamos en parágrafos anteriores sobre la configuración del espacio latinoamericano. Esta distinción es importante porque lleva a algunos autores a plantear el giro de la mirada de la PEA hacia la región o el latinoamericanismo que esta lleva adelante, sin considerar su interacción con actores fuera de la subregión. Así, por ejemplo, en el caso que estamos estudiando, nos llevaría a conclusiones contradictorias pensar que Argentina es latinoamericanista y sin embargo tiene acercamientos esporádicos con México que no tienen la misma jerarquía o densidad que el vínculo con, por ejemplo, los países del Mercosur. Consideramos que esta definición es contraproducente para el análisis, ya que soslaya aspectos relevantes de la proyección internacional del país.

Otra visión puede ser aquella que se pregunta si la PEA jerarquiza las relaciones tanto bilaterales con los distintos países de la región más allá de Sudamérica. A esta cuestión, Bueno (2014) responde que en realidad existe una "búsqueda intermitente de protagonismo subregional complementado con cierto acercamiento hacia México y especialmente a Venezuela". Esto no significa que las relaciones tanto con México como con el resto de los países latinoamericanos sean desdeñables. Por ejemplo, como mencionábamos al principio del presente parágrafo, aunque solo fuera como "acumulación" de apoyos para su posición respecto a la cuestión Malvinas, la diplomacia y la presidenta argentina se ocuparon de destacar en diversos ámbitos los respaldos obtenidos por los distintos países de América Latina.

Desde esta perspectiva, no es necesario buscar en la PEA una estrategia política para América Latina en su conjunto, sino que es importante analizar los lazos bilaterales y las relaciones de poder que se van configurando en este espacio. Así, surge una temática que menciona Bueno (2014): la posibilidad del acercamiento argentino hacia México para equilibrar el peso de Brasil. Esto es interesante por un lado porque propondría a un socio latinoamericano "del Norte" estratégico para la configuración de poder en América Latina del Sur pero también de la región en su conjunto.

Esto nos lleva a dos temas. Por un lado, la estrategia argentina de apoyo al grupo conformado, entre otros, por México para la propuesta de reforma del Consejo de Seguridad de Naciones Unidas. Argentina apostó así por un proyecto que amplíe el número de asientos en el órgano y les otorgue mayor representatividad regional, sin por 


\section{LA INCIDENCIA DE LAS VARIABLES PERSISTENTES DE LA POLÍTICA EXTERIOR ARGENTINA: EL CASO DE LA RELACIÓN CON MÉXICO}

\section{MARÍA JULIA FRANCÉS}

ello aumentar el número de miembros permanentes. Así, junto con México, Argentina se opone a la posición de Brasil, que busca obtener una banca permanente con todos los derechos que ello implica. Brasilia de esta manera buscaría erguirse como "representante" de los intereses de la región, mientras que México y Argentina proponen una representación por votación, más democrática y con capacidad para llevar adelante gestiones a más largo plazo. De esta manera, Argentina tomó en cuenta la importancia que revisten para ella estos aspectos regionales y su vínculo con Brasil y halló coincidencia de intereses con México. Por otra parte, algunos autores vieron en el Acuerdo de Asociación Estratégica entre Argentina y México un paso en la dirección correcta para la concreción del potencial que tendría el segundo para contrabalancear el poderío de la nación lusófona y consolidar una verdadera PEA latinoamericanista ${ }^{\mathrm{XVII}}$. A pesar del entusiasmo inicial, no vemos que esto se haya logrado verdaderamente.

Continuando con esta perspectiva, un hecho significativo durante el período bajo estudio fue la destitución del presidente hondureño Manuel Zelaya. Nos interesa este tema porque se trata de un incidente acaecido en una zona prioritaria para la política exterior mexicana que, sin embargo, no reviste tal carácter dentro de la PEA. El hecho fue relevante para la Argentina ya que puso en juego una serie de principios y valores que se había planteado como prioritarios para su accionar externo, en particular aquellos vinculados con los derechos humanos y la defensa de la democracia.

Así, luego de que el 28 de junio de 2009 Roberto Micheletti expulsara al mandatario de su país, la Argentina se unió al pedido de distintos países de la región y fuera de ella para que se restituyera al jefe de Estado y rechazó de plano la legitimidad de lo que consideró un "golpe blando". Este posicionamiento argentino llevó al país a acercar su postura a la de un socio estratégico en la zona: México. La situación era de particular interés para la nación norteamericana ya que puso en juego un objetivo de su política exterior, el liderazgo, así como su interés por la estabilidad del istmo y por ganar prestigio internacional a través de su rol en el conflicto ${ }^{\text {XVIII }}$. Mientras tanto, para la PEA, según Miranda (2010:6), este conflictos más allá de Sudamérica era una posibilidad de plasmar un "ascendiente diferenciador" que la proyectara hacia el norte y demostrar su capacidad de jugar un papel trascendente a nivel regional que reforzara su posición en el cuadro de las relaciones tanto sudamericanas como hemisféricas.

Este hecho incidió en la relación con México de diversas maneras. Por un lado, en ámbitos multilaterales y regionales, como la CELAC, gestionaron junto a los demás miembros una respuesta inicial conjunta en poco tiempo ${ }^{\text {XIX }}$. Además, en esta primera etapa, el golpe se constituyó en un tema de la agenda que ocupó al canciller argentino en su misión política y comercial hacia México en agosto de 2009. La diplomacia argentina declaró tener una posición común con el gobierno de Calderón (La República, 20/08/2009) y colocó la cuestión en el centro de los debates en distintos foros compartidos por ambos países ${ }^{\mathrm{XXX}}$. Mientras tanto, en una segunda etapa, tras las elecciones en Honduras y el acuerdo de Cartagena, Argentina se plegó a una posición 


\section{LA INCIDENCIA DE LAS VARIABLES PERSISTENTES DE LA POLÍTICA EXTERIOR ARGENTINA: EL CASO DE LA RELACIÓN CON MÉXICO}

\section{MARÍA JULIA FRANCÉS}

similar a la que venía esbozándose ya en la actitud mexicana y que había sido criticada por Hugo Chávez (García, 08/12/2009): admitir la relevancia del sufragio y terminar por reestablecer las relaciones diplomáticas con Tegucigalpa. Para Miranda (2010:6), el rol que jugó Argentina en esta situación fue una muestra clave de "presencia oportuna pero exigua" en conflictos a nivel latinoamericano y reflejó el juego argentino de dualidad cercanía - lejanía respecto a los países de dicha región. Esto nos lleva a pensar que Argentina podría haber llevado adelante una política latinoamericanista más vigorosa para la defensa de la democracia si hubiera logrado constituirse en socio de México para la gestión de la crisis. Sin embargo, quedó eclipsada por el papel jugado por Brasil, país al que Calderón priorizó para la búsqueda de coincidencias, según Soriano (2010).

Este tipo de latinoamericanismo es también compatible con una estrategia argentina de búsqueda de protagonismo en su relación con otros actores de la región por antagonismo con EEUU o con intenciones autonomizantes respecto a potencias extrarregionales. Así, esta variable puede ser considerada también como un integrante más en la tensión de las relaciones con Estados Unidos y Europa ${ }^{\mathrm{XXX}}$.

Por último, consideramos pertinente mencionar otra interpretación de latinoamericanismo. En este caso, se trata del rol que le cabe a la región en su conjunto dentro de la PEA. Esta acepción le da importancia a los aspectos multilaterales, así como a las referencias simbólicas y al problema de la "pertenencia". Esto significa que, más allá de las relaciones bilaterales, la relacionamos con la evocación de un sentimiento de confraternidad en este espacio y la búsqueda de políticas colectivas comunes que superen los particularismos. De existir esta tendencia, podría influir en la PEA hacia México al enmarcarla dentro de una posible estrategia general y una jerarquía particular de la región. Interpretada de esta manera, esta variable puede incidir en el modo en que México y Argentina se relacionan, pero el mayor acercamiento o alejamiento en el caso particular de México determina la existencia de una PEA latinoamericanista.

Aquí nos interesa retomar las consideraciones de Bueno (2014) quien plantea que las últimas administraciones en Argentina rompen con la tendencia que encontraba Russell a lo largo de la historia del país a denostar este vínculo y plantear que argentina es solo "accidente geográfico". Los funcionarios argentinos durante el período remarcaron pertenencia cultural, histórica, idiomática y hasta política a la región e hicieron frecuentes referencias en su discurso a la búsqueda de estrategias comunes, problemas comunes y la necesidad de concertación.

En este sentido, nos interesa mencionar las instancias de cooperación política y técnica compartidas por Argentina y México. Tanto el Acuerdo de Complementación Estratégica como distintos acuerdos de cooperación y los comunicados emitidos luego de reuniones bilaterales ambos mencionan la relevancia de su identidad compartida como latinoamericanos y su intención de formular proyectos y estrategias comunes hacia la región. Si bien al tratarse de documentos conjuntos es difícil establecer si este 


\section{LA INCIDENCIA DE LAS VARIABLES PERSISTENTES DE LA POLÍTICA EXTERIOR ARGENTINA: EL CASO DE LA RELACIÓN CON MÉXICO}

\section{MARÍA JULIA FRANCÉS}

hecho se enmarcó en una política argentina general o si, por ejemplo, se incluyó la cuestión por iniciativa mexicana, lo que queda claro es que la PEA ya no desdeña su pertenencia a la región.

Las referencias a la región en su conjunto deben interpretarse también a la luz de los cambios en la política exterior mexicana que, con Felipe Calderón y Peña Nieto, fue volviendo la mirada hacia América Latina, aunque priorizando en particular la relación

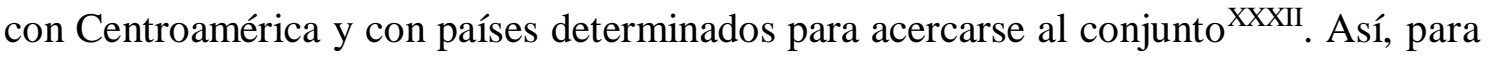
la política exterior argentina, hablar de América Latina en sus instancias de cooperación y diálogo con México implica la búsqueda de un marco de referencia común que, como vimos, no es tal necesariamente, pero también podría ser una oportunidad para volver la mirada hacia áreas de la región más allá del Cono Sur, en especial el istmo centroamericano $^{\mathrm{XXXIII}}$.

Si hablamos del vínculo de la Argentina con el conjunto de la región, podemos mencionar su participación tradicional en mecanismos como la Asociación Latinoamericana de Integración (ALADI), así como el grupo de Río (y a partir de 2010, la Comunidad de Estados Latinoamericanos y Caribeños -CELAC) y las Cumbres Iberoamericanas. En este marco, Argentina encontró posibilidades de acercamiento y concertación con México. Respecto a la importancia de los ámbitos multilaterales de este tipo y la PEA hacia la región, Flemes y Wehner (2013) plantean, por ejemplo, que Argentina llevó adelante una estrategia a través de la CELAC para atraer a México a la América Latina e incorporarlo a las políticas regionales, según los autores, para contrarrestar el peso de Brasil.

A pesar de las consideraciones anteriores, coincidimos con Miranda (2013) en que la PEA demostró una tenencia mucho más sudamericanista que latinoamericanista en este período, ya que priorizó los lazos con sus vecinos más cercanos y, en particular, con Brasil. Las referencias discursivas a la región no ocultaron la predominancia del vínculo con la subregión y los acercamientos esporádicos a países como México no parecieron ser parte de una estrategia sistemática de PEA para el conjunto de América Latina tanto en su aspecto bilateral como multilateral.

\section{Conclusiones}

Del análisis realizado podemos realizar una serie de conclusiones. En primer lugar, vemos que efectivamente las variables persistentes de la política exterior argentina tuvieron una incidencia en algunos casos positiva y en otros, negativa, sobre la vinculación de este país con México. Nuestro interés por este caso particular nació de las consideraciones acerca de la configuración de dos subregiones cada vez más diferenciadas dentro de la región latinoamericana. Buscábamos comprender cómo se comportaría la PEA ante esta "lejanía" y, en el marco de este nuevo escenario, qué sentido tuvo la variable "vínculo con la región". Para ello, analizamos la dimensión 


\section{LA INCIDENCIA DE LAS VARIABLES PERSISTENTES DE LA POLÍTICA EXTERIOR ARGENTINA: EL CASO DE LA RELACIÓN CON MÉXICO}

\section{MARÍA JULIA FRANCÉS}

económica de la relación bilateral y vimos cómo la priorización por parte de la política argentina de sus intereses comerciales tuvo consecuencias negativas para los lazos con México. Afirmamos esto porque redujo variedad real de temáticas que constituyeron la agenda bilateral a un aspecto que generó enfrentamientos y tensiones, dadas las diferencias en los principios esgrimidos por ambos. Es verdad que Argentina demostró que le resultaba deseable una superación de estos conflictos y buscó darles respuestas pragmáticas. Pero evaluamos que podría haber sido más productivo sostener instancias de concertación política, en el marco, por ejemplo, de su Acuerdo de Asociación Estratégica, para evitar el enfriamiento de la relación. Así, una jerarquización inversa a la evidenciada, es decir la subordinación de los aspectos económicos a la dimensión política de la PEA, podría haber fomentado mayores entendimientos entre ambos socios.

En la dimensión económica, sin embargo, pudimos ver también la incidencia de otras variables persistentes, como el juridicismo y el latinoamericanismo, que moldearon sus consecuencias de una manera que consideramos positiva. Así, la jerarquía para la agenda de PEA que tuvieron las cuestiones territoriales y de recursos naturales, así como la incidencia del juridicismo en la relación bilateral, generó dinámicas que consideramos positivas para el mayor desarrollo del vínculo. Decimos esto porque creemos, por ejemplo, que el impulso dado por la administración de Fernández al tratamiento de la cuestión Malvinas en foros internacionales y en distintas instancias diplomáticas, más allá de los debates de fondo, permitió una serie de coincidencias entre la Argentina y México que, frente a las disidencias en otros ámbitos, pueden ser valiosas para el vínculo.

En cuanto a la jerarquía de la región en la PEA, vimos que distintos autores interpretan de distintas maneras este concepto y que durante el período estudiado, el hecho de que Argentina priorizara sus vínculos bilaterales con algunos países específicos de Sudamérica tuvo consecuencias negativas para su relación con México. En este sentido, una PEA con la mirada puesta en la subregión hizo que México cumpliera un rol secundario y esporádico en su estrategia internacional, supeditado en algunos casos, a la relación con Brasil. Sin embargo, rescatamos la reivindicación retórica de la Argentina en referencia a su pertenencia a Latinoamérica, a diferencia de otras etapas históricas. La consecuente participación en foros regionales y su presencia, aunque exigua, en cuestiones de interés regional (como el golpe de Estado en Honduras) pueden tener una incidencia positiva sobre el vínculo con México al fomentar espacios de debate y concertación compartidos. Finalmente, consideramos que la combinación del latinoamericanismo con la variable vínculo con Estados Unidos/Europa puede tener un efecto negativo sobre la agenda con México y terminar por generar más tensiones que coincidencias.

Consideramos que si la Argentina tuviera una estrategia coherente y a largo plazo hacia la región en su conjunto y con sus partes de manera bilateral, superando la 


\section{LA INCIDENCIA DE LAS VARIABLES PERSISTENTES DE LA POLÍTICA EXTERIOR ARGENTINA: EL CASO DE LA RELACIÓN CON MÉXICO}

\section{MARÍA JULIA FRANCÉS}

visión economicista, esta podría robustecer su vínculo con México y lograr una agenda más compleja y variada que podría beneficiar a ambos.

\section{Notas}

' Graduada del programa de postgrado en Cooperación Cultural Internacional de la Universidad de Barcelona, traductora por el Instituto de Educación Superior Olga Cossettini, postitulada en la Universidad Nacional de Rosario, editora responsable del Observatorio de Política Exterior Argentina.

${ }^{\text {II Lasagna, } 1996 .}$

III Lasagna, 1996.

IV Soriano, 2013.

${ }^{\mathrm{v}}$ Bueno, 2012.

${ }^{\mathrm{VI}}$ Bueno, 2014.

VII Bueno, 2012.

VIII Yankelevich, 2000.

Ix Miranda, 2004: 134,

x Miranda, 2010: 2.

XI Lasagna, 1996.

XII Miranda, 2011.

XIII Miranda, 2011.

XIv Rincón, 2000.

${ }^{\mathrm{XV}}$ Soriano, 2007.

${ }^{X V I}$ Ver referencia a variables persistentes en Bueno (2012).

XVII Francés, 2013.

XVIII Soriano, 2013.

XIX "En mayo de 2011 inauguró en Veracruz un centro de entrenamiento y capacitación, así como una nueva planta con capacidad de producción de 500 mil toneladas anuales de tubos sin costura de 2.4 a 7 pulgadas de diámetro. Además, a principios de 2012 iniciaron, en instalaciones en este estado, la producción de varillas de bombeo luego de una inversión inicial de 35 mdd. La capacidad, para 2013, será de un millón de este tipo de varillas al año".

xx Entre las empresas mexicanas con inversión en Argentina destacan Coca-Cola FEMSA en la rama de bebidas, América Móvil en telecomunicaciones, CIE en entretenimiento, Bimbo en alimentos, ICA en construcción, Grupo Posadas en hotelería, Mabe en electrodomésticos, Alsea en restaurantes y Xignux en el sector automotriz".

XxI Fajardo, Carabajal Suárez, 2012.

xxII Fajardo, Carabajal Suárez, 2012.

xxIII Esto incluyó el convenio de colaboración firmado entre Cámara de Comercio Mexicano Argentina, A.C. (CCMA) y la Asociación de Importadores y Exportadores de la República Mexicana, A.C. (ANIERM), en busca de nivelar la balanza comercial entre ambos países (Embajada Argentina 2012).

xxIV Lo que es trascendente es que la República Argentina se halla en proceso de negociación precisamente con la República Federativa de Brasil, en un acuerdo para el fomento de la integración productiva con estímulos para la producción de autopartes, y también integrar junto a México toda una plataforma de producción y desarrollo de la industria. Esto significa también el mantenimiento de estándares altos de calidad y tecnología a nivel mundial, generando la calidad del producto y el cuidado de las personas y el medio ambiente.

XXv Bueno, 2014.

Xxvi La Gaceta Tucumán, sábado 07 de septiembre de 2013.

XXVII Bueno, 2014.

XXVIII Soriano, 2009.

XxIX Soriano, 2010.

Cadernos do Tempo Presente, n. 24, jun./jul. 2016, p. 136-161| http://www.seer.ufs.br/index.php/tempo 
xxx Francés, 2013.

XXXI Bueno, 2014:14.

XXXII Soriano, 2013.

XXXIII En cuanto a la cooperación técnica, por ejemplo, Centroamérica es un área prioritaria para la política exterior mexicana, mientras que para la PEA no tiene la misma jerarquía, aunque no es desdeñable. Al mismo tiempo, México y Argentina han logrado diversos acuerdos de ayuda mutua y, cuantitativamente al menos, han logrado colocarse mutuamente entre sus principales socios cooperativos. Estos proyectos revisten características particulares en especial en cuanto a la reciprocidad y han llevado a que ambos expresen su deseo de trabajar en conjunto para cooperar con terceros países. En un futuro, esta sería una posibilidad para la Argentina de realizar una estrategia cooperativa verdaderamente regional, superando su actual visión centrada en sus vecinos.

\section{Referencia Bibliográfica}

\section{Artículos especializados}

BONAVITTA, Paola (2011), "Golpe de Estado en Honduras: el discurso de Cristina Fernández", revista Razón y Palabra, NÚMERO 74, NOVIEMBRE 2010 a ENERO de 2011, disponible en: www.razonypalabra.org.mx

BUENO, María del Pilar (2012), "Argentina: entre la decepción y la esperanza. Un breve análisis de la política exterior 2003-2012", Mesa Redonda para el X Congreso Nacional y III Congreso Internacional sobre Democracia, Facultad de Ciencia Política y Relaciones Internacionales de la Universidad Nacional de Rosario.

BUENO, María del Pilar (2014) "Autonomía, tendencias profundas y variables persistentes de la Política Exterior Argentina (2003-2012)", en Brazilian Journal of International Relations, vol. 3, núm. 2.

FAJARDO MORALES, María Esther y CARABAJAL SUÁREZ, Yolanda (2012), "La pérdida de un eslabón más: el conflicto automotriz entre Argentina y México", Revista trimestral de Análisis de Coyuntura Económica, julio-septiembre, vol. V, número 3, México.

FLEMES, Daniel, WEHNER, Leslie (2013) "Reacciones estrategicas en Sudamerica ante el ascenso de Brasil", Foreign Affairs Latinoamerica, Vol. 13: Num 4, pp 107114 , disponible en www.fal.itam.mx

LASAGNA, Marcelo (1996), "Cambio institucional y política exterior: un modelo explicativo", en revista Afers Internacionals, núm. 32, Fundación CIDOB. 
MIRANDA, Roberto (2010), "La política regional de Argentina: Del latinoamericanismo al sudamericanismo", ponencia en el V Congreso de Relaciones Internacionales, Universidad Nacional de La Plata, La Plata, disponible en: www.iri.edu.ar.

MIRANDA, Roberto (2004), "Argentina y la política latinoamericana: la cuestión de las diferencias", en revista Relaciones Internacionales - número 27.

PROMÉXICO (2011), Síntesis de la Relación Comercial México-Argentina, Unidad de Inteligencia de Negocios, documento del 10 de mayo, México.

RUSSELL, Roberto (1990) "El proceso de tma de decisiones en la Política Exterior Argentina (1976-1989)", en RUSSELL, Roberto (ed.), Política Exterior y toma de decisiones en América Latina, Buenos Aires, GEL .

SORIANO, Juan Pablo (2010), "México ante la crisis hondureña: estabilidad regional y prestigio internacional", revista Opinión América Latina, núm. 47, mes de julio, Barcelona.

YANKELEVICH, Pablo (2000), "Políticas, negocios y poetas. Un rastreo en los orígenes de las relaciones entre México y Argentina" en México-Mercosur: un enfoque desde la relación México-Argentina, ed. Plaza y Valdés, México.

\section{Artículos periodísticos y comunicados de prensa}

América Economía, "Presidenta argentina agradece a Peña Nieto rol de Pemex en acuerdo con Repsol", jueves 28 de noviembre de 2013, disponible en: http://www.americaeconomia.com/node/105848

Cámara De Comercio Mexicano-Argentina, "Comercio bilateral México-Argentina 2002-2012", Documento de trabajo, mayo de 2013.

Comunicado de Embajada Argentina en México, "Se realizó en México encuentro histórico por la Cuestión Malvinas", México, 19 de junio de 2013, disponible en: http://www.embajadaargentina.mx/noticia.php?id=1173

García, Jacobo (2009) "Hugo Chávez en contra de México por su actitud frente a Honduras", diario El Mundo, 08/12/2009, disponible en: www.elmundo.es 
International Center for Trade and Sustainable Development (2012) "México retira solicitud de panel por medidas de importación de Argentina, mientras que Panamá inicia su propio caso", Bridges Weekly Trade News Digest, Vol. 16, No. 44, 19 de diciembre de 2012.

Mercopress, "YPF and Repsol are expected to sign a compensation deal next week", sábado 22 de febrero de 2014, Montevideo, disponible en: http://en.mercopress.com/2014/02/22/ypf-and-repsol-are-expected-to-sign-acompensation-deal-next-week

Página 12, "Giorgi: México retirará la demanda contra la Argentina en la OMC", sección Últimas Noticias, viernes 14 de diciembre de 2012, disponible en: http://www.pagina12.com.ar/diario/ultimas/20-209879-2012-12-14.html

Presidencia de la Nación, "El Jefe de Gabinete de Ministros puso en funcionamiento Programa de Aumentos y Diversificación de Exportaciones ", sección Últimas Noticias, visitado el 20 de junio de 2014. Disponible en: http://www.presidencia.gov.ar/component/content/article/141-ultimas-noticias/27043el-jefe-de-gabinete-de-ministros-puso-en-funcionamiento-programa-de-aumentos-ydiversificacion-de-exportaciones 